This is the author version of the following article:

Podlaskowski, A. (2012). "Simple Tasks, Abstractions, And Semantic

Dispositionalism." Dialectica, December, 66 (4), pp. 453-470, which has

been published in final form at:

http://onlinelibrary.wiley.com/doi/10.1111/1746-

8361.12001/abstract;jsessionid=639DB8C7798B8AA60ACA8FE66540F867.f03t01 globalMessage $=0$

\title{
Simple tasks, abstractions, and semantic dispositionalism
}

\author{
Adam C. Podlaskowski ${ }^{\dagger}$
}

\begin{abstract}
According to certain kinds of semantic dispositionalism, what an agent means by her words is grounded by her dispositions to complete simple tasks. This sort of position is often thought to avoid the finitude problem raised by Kripke against simpler forms of dispositionalism. The traditional objection is that, since words possess indefinite (or infinite) extensions, and our dispositions to use words are only finite, those dispositions prove inadequate to serve as ground for what we mean by our words. I argue that, even if such positions (emphasizing simple tasks) avoid the traditional form of Kripke's charge, they still succumb to special cases of the finitude problem. Furthermore, I show how such positions can be augmented so as to avoid even these special cases. Doing so requires qualifying the dispositions of interest as those possessed by the abstracted version of an actual agent (in contrast to, say, an idealized version of the agent). In addition to avoiding the finitude problem in its various forms, the position that results provides new materials for appreciating the role that abstracting models can play for a dispositionalist theory of meaning.
\end{abstract}

\section{Introduction}

According to semantic dispositionalism, the meanings of the terms an agent uses are determined by some of her dispositions, such as those of a recognitional, discriminatory, or inferential variety. As part of his case for meaning

\footnotetext{
${ }^{\dagger}$ College of Liberal Arts, Fairmont State University, 1201 Locust Avenue, Fairmont WV 26554-2470; Email: apodlaskowski@fairmontstate.edu
} 
scepticism, Saul Kripke (1982) offers several criticisms of this kind of position. Among them is what will be called the finitude problem: while terms possess indefinite (or even infinite) extensions, agents like us possess only finite dispositions. So we cannot, contrary to the ambitions of the semantic dispositionalist, ground the intended meaning of terms on the basis of such dispositions. ${ }^{1}$

In reaction to this problem, Simon Blackburn (1984) and Neil Tennant (1997) (building on a suggestion by Jon Cogburn) have denied that we should appeal to the total dispositional state of an agent in order to settle what she means by her words. ${ }^{2}$ Rather, they suggest that a finite agent's ability to indefinitely apply a term is best explained by appeal to her dispositions to complete simple, entirely surveyable tasks. Here, I argue (in $\S 4.1$ and $\S 4.2)$ that, despite the appeal of this position, it still faces special cases of the finitude problem. Furthermore, I show how the use of abstracting models can remedy these problems. As Nicholaos Jones and I originally argued, the traditional finitude problem can be solved by appealing to the dispositions possessed by the abstracted version of an agent, where the limits on the traits responsible for our finite nature are ignored in a principled fashion (Podlaskowski and Jones, 2012). I adapt a similar strategy here to the Blackburn-Tennant-Cogburn approach (in $\S 5, \S 5.2, \S 5.3$, and $\S 5.4$ ), allowing us to keep some of what is promising about the approach.

Of course, the initial appeal of the Blackburn-Tennant-Cogburn approach is its promise to resolve the finitude problem. So, it is reasonable to wonder whether the Blackburn-Tennant-Cogburn approach retains any of that appeal if it must rely on abstracting models in order to avoid special cases of the finitude problem. To address this concern, it is established that the Podlaskowski-Jones approach also benefits from its merging with the Blackburn-Tennant-Cogburn approach; as such, the latter approach takes on a new significance, still in relation to the finitude problem. More specifically, while the case that Jones and I originally made for the use of abstracting models was in reaction to one of Kripke's charges against the use of idealizing models, Kripke raised an additional charge that we did not explicitly

\footnotetext{
${ }^{1}$ This problem is generalizable to any attempt to (metaphysically) reduce meaning to naturalistically respectable materials (including physical states, capacities, or abilities, all of which might be finite). Solutions (to the finitude problem for physical states, capacities, or abilities) can be offered analogous to those addressed in this article.

${ }^{2}$ Though Tennant developed the position in some detail, he credits Cogburn with originally suggesting the strategy $(1997,153$, note 6$)$.
} 
address. A version of that charge, adapted to the use of abstractions, is raised (in $\S 6, \S 6.1$, and $\S 6.2$ ); and it is shown, by citing the dispositions emphasized in the Blackburn-Tennant-Cogburn approach, that an apt reply is made available.

In short, this essay is an exercise in symbiosis, executed on behalf of two forms of semantic dispositionalism. As a preliminary step to making this case, a basic characterization is given (in the next section) of meaning skepticism and the finitude problem facing semantic dispositionalism.

\section{The traditional finitude problem}

With the sceptical paradox he attributes to Wittgenstein, Kripke (1982) threatens many theories of meaning, including semantic dispositionalism. To illustrate the challenge, consider an agent's use of the symbol ' + '. Suppose she were asked to answer ' $25+200=$ ?'. If she meant addition by ' + ', she should answer ' 225 '. The meaning sceptic, though, demands that we cite the fact about this agent in virtue of which she means (or meant) addition instead of some other function, e.g., quaddition, where one has successfully quadded just in case, if both of the numbers (presented) are less than 57, one should produce their sum; and if either of the numbers (presented) is greater than or equal to 57, one should answer ' 5 '. The semantic dispositionalist answers the meaning sceptic by appealing to an agent's dispositions to use the symbol ' + '. According to one version of the thesis, that an agent means addition by the use of the symbol ' + ' is owing to her being disposed, under conditions that are normal, standard, or favorable, to answer ' 225 ' to the question ' $25+200=$ ?' and to give answers indicative of addition for all other pairs of numbers. ${ }^{3}$

This sort of answer to the meaning sceptic presents a problem: although

\footnotetext{
${ }^{3}$ The appeal to standard, optimal, or favorable conditions is an attempt to differentiate between those dispositions that ground correct uses of expressions from those that ground incorrect uses. It has been argued, however, that any such appeal must be viciously circular. For related arguments, see Boghossian (1989, 537-540) and Fodor (1990, 5982). For a reply to Boghossian's (1989) objection from semantic holism, see Podlaskowski (2010). And for an argument that semantic holism actually aids the opponent of the meaning sceptic, see Tennant (1997, 108-15). For more general defenses of the appeal to conditions of the, e.g., standard, favorable, or optimal variety, see Miller (1997) and Pettit (1999). As to whether this sort of strategy - or another one - allows for successfully distinguishes between correct and incorrect uses of terms is not an immediate concern.
} 
there are infinitely many numbers that might be added together, ordinary agents are not disposed to do so for any two numbers, because most numbers are too large for ordinary agents to process. This point generalizes easily: whereas any term can be applied correctly an indefinite (or even infinite) number of times, the dispositions of an agent like us are only finite. The correct application of any given term outpaces the dispositions of finite agents like us, therefore rendering those dispositions inadequate for reading an agent's intended meaning off her dispositions. Again, call this the finitude problem for semantic dispositionalism.

\section{Simple tasks and semantic dispositionalism}

In reaction to the finitude problem, Tennant identifies and rejects an assumption made by the meaning sceptic as unduly strong:

Why should one accept the implicit assumption that the dispositionalist is committed to having the subject be able to carry out every command to add two numbers, no matter how large? Clearly, this is too extravagant a demand. Why not rather say that the way to check a subject's 'correct' dispositions with regard to addition is to present him with any (stretch of) a computation of the sum of two numbers, and then, with respect to any surveyable aspect of such a computation, ask him whether it is in order? $(1997,138)$

In place of the meaning sceptic's presumption that we appeal to the total dispositional state of an agent to use a particular expression, Tennant suggests that an agent need only possess the factorizable dispositions to handle a localized segment of a calculation or proof $(1997,139) .{ }^{4}$

To illustrate these dispositions, consider the following fragment of an addition problem:

$$
\begin{array}{r}
1 \\
\ldots 67 \ldots \\
+\ldots 15 \ldots \\
\hline \ldots 82 \ldots
\end{array}
$$

\footnotetext{
${ }^{4}$ Though Tennant's (1997) point is part of a larger semantic anti-realist framework, it has intuitive appeal independent of that project.
} 
So long as one can add together ' 7 ' and ' 5 ', carry the ' 1 ', and add together ' 6 ', ' 1 ', and the carried over ' 1 ' - without regard to the particular place that these columns occupy within the larger addition problem - we can say the relevant competencies are in place. Competence with this method depends on one's disposition to handle any given column of an addition problem in decimal notation, as well as how these basic tasks are combined. For instance, such an agent is disposed to carry numbers from one column to another, to recognize which side of the problem with which to begin one's calculations (e.g., the right side in the right-to-left method), and to recognize what counts as the terminating point of a calculation (e.g., in the right-to-left method, when we run out of numbers to add together on the left side of the problem).

That the finitude problem is avoided depends on emphasizing that meaning is fixed by an agent's factorizable dispositions left unthreatened by the meaning sceptic's attacks. With something as simple as the disposition to consult a look-up table for the addition of single digits, the number of available possibilities does not serve the meaning sceptic's purposes. After all, the finitude problem is motivated by raising possible uses of a term beyond one's finite capabilities; however, on the present approach, the relevant possibilities appear constrained to dispositions to use a completely surveyable look-up table. We can appeal to these dispositions as the metaphorical building blocks (hence "factorizable") from which any application of a term is constituted; the determinacy enjoyed by our factorizable dispositions is transferred to more complex configurations of those dispositions.

A similar reply to the finitude problem is offered by Blackburn, who suggests that one's intending to mean addition by ' + ' is established by the fact that, for a question such as ' $\mathrm{x}+\mathrm{y}=$ ?':

The answer I would accept is the one that would be given by reiterating procedures I am disposed to use a number of times.... The equation would be: By '+', I mean that function $F$, that accords with my extended dispositions if and only if (i) it is the answer I am disposed to give and retain after investigation, or (ii) it is the answer I would accept if I repeated a number of times procedures I am disposed to use, this being independent of whether I am disposed to repeat those procedures that number of times. (1984, 289-290)

We might read Blackburn's description of one's extended dispositions as relying on a distinction between one's disposition to use a procedure and the 
opportunities to manifest that disposition at different times and in different numbers of repetitions. ${ }^{5}$ The indefinitely many applications of a term derive, in part, from the indefinitely many occasions for manifesting the relevant dispositions. As such, the emphasis on one's extended dispositions, along with the fact that there are indefinitely many situations in which they might manifest, is meant to show that one's finite dispositions do not engender the finitude problem, as Kripke insists. Much as for Tennant's account, Blackburn maintains that the dispositions to complete simple tasks which actual agents possess - properly understood - provide the basis for avoiding the traditional form of the finitude problem. ${ }^{6}$

\section{Special cases of the finitude problem}

Though an attractive position, the Blackburn-Tennant-Cogburn approach is not without problems. As argued in the next two sections ( $\S 4.1$ and $\S 4.2$ ), the position is subject to two special cases of the finitude problem.

\subsection{The combination problem}

First, there is what will be called the combination problem. The problem arises because the Blackburn-Tennant-Cogburn approach emphasizes both dispositions to complete simple tasks and dispositions to coordinate, order, or combine those simple tasks in the right way. While both sorts of dispositions

\footnotetext{
${ }^{5}$ This interpretation is similar to the one given by Jussi Haukioja (2004). Blackburn's position, so understood, resembles somewhat the account provided by Martin and Heil (1998, 297-304), who argue that a disposition should not be confused with the opportunities for its manifestation; though a being might possess a disposition which is finitely manifestable, this does not mean that the disposition itself is finite.

${ }^{6}$ While the discussion has been restricted to examples of logical and mathematical terms, it is worth considering if these solutions apply to any sort of term. Settling the matter depends on the details of the positions. Tennant (1997) is well placed to provide a general account, for he applies his point about factorizable dispositions to the rules operative in any fragment of a proof, and argues that such rules provide the basis for a general account of meaning. Whether Blackburn's approach (1984) can be generalized to any sort of term has not been demonstrated. However, there is no obvious obstacle to doing so. But if the account fails to apply to every sort of expression, Blackburn's account is not alone, for as Mark Sprevak observes (2008, 285-6), many notable solutions to meaning scepticism also apply only to restricted classes of expressions.
} 
are factorizable, it is doubtful (given our finite limits) that we possess the latter dispositions to the extent required to fix what we mean by our words.

In order to state the combination problem for the case of addition, first consider the sorts of dispositions targeted. When using the right-to-left method of adding, one should (for instance) handle each column in the same fashion, moving from one column to the next left adjacent column in the fashion prescribed by the method. One should start with the right-most column first, and one may not whimsically jump around from column-to-column. Call these sorts of dispositions combinatory dispositions. Now consider an addition problem involving too many columns to be solved in one's lifetimea case involving many iterations of the same combinatory dispositions. Even if we are disposed to provide the relevant sum for each column handled, and then continue to the left adjacent column, we have our limits: we can only complete the same simple task in a particular order so many times. This proves problematic because adding requires handing columns in a particular order; and so our inability to handle each column in the appropriate order invites indeterminacy regarding our use of ' + '.

Keeping with the emphasis on factorizable dispositions, it is tempting to say that we possess the same disposition to move from the tenth column to the eleventh column of an addition problem as we do moving from the onetrillionth column of an addition problem. This temptation should be resisted, though, as such a picture remains insensitive to the fact that, as adders, we ought to proceed in a particular order - a fact that surely affects how we characterize the relevant dispositions. To better see this, imagine the difficulties one would face trying to coordinate one's activities (producing sums within columns) when faced with solving an addition problem involving, say, ten columns, were one's attention to wander from moment-to-moment and one possessed next to no working memory. The result would be someone who is arithmetically adrift: though perhaps capable of producing the sum for the numbers in any given column, such an unfortunate figure would have no assurance of coordinating one's activities in the manner of adding. By magnifying that situation to the case of an addition problem involving an enormous number of columns, the claim that we possess the required combinatory dispositions for any given column in any given addition problem becomes highly dubious. So, contrary to Tennant's insistence that competence with addition involves the disposition to handle any arbitrary column of an addition problem, competence with addition involves the disposition to handle columns in a particular order. Despite possessing the relevant fac- 
torizable dispositions, then, our finite limits block possessing them for any given column in the order required by addition. Hence, the meaning sceptic gains a new foothold.

\subsection{The input problem}

The second problem facing the Blackburn-Tennant-Cogburn approach is what will be called the input problem. The problem is that there are terms whose applications cannot be broken down into tasks involving manageable inputs of the sort required by the Blackburn-Tennant-Cogburn approach.

This concern does not arise for every term. For the approach's favored case of addition, the sorts of factorizable dispositions to which Tennant and Cogburn appeal are well-suited (combination problem, aside). The same cannot be said, however, for the factorizable dispositions fixing that, by the word 'and', we mean the conjunction operator found in a natural deduction system. ${ }^{7}$ To help see this, suppose that one's grasp of conjunction is determined by one's grasp of the corresponding introduction rule ("From a sentence $p$ and a sentence $q$, we may infer $p$ and $q$ ") and elimination rule ("From a sentence $p$ and $q$, we may infer $p$, as well as $q ")$. Notice also that the set of proofs featuring those rules is infinite, that the proofs themselves can be of any finite length, and that those proofs get larger (in two dimensions) as the sentences themselves get longer. This proves problematic because the use of an introduction or elimination rule on some enormously long conjunction involves at least one enormously long conjunct, and there is no way to factor out anything sufficiently small for a finite agent like us to manage. That is, there are enormously long sentences for which we lack the memory, lifespan, etc. needed in order to conjoin it with any other sentence in the manner of the conjunction introduction rule; and for a conjunction including an enormously long conjunct, we lack the memory, lifespan, etc. required to apply the elimination rule. So, appealing to an agent's dispositions to complete simple tasks fails to ground any given use of the word 'and'. While there is clearly a procedure in place for deciding whether those rules have been applied correctly or incorrectly, the problem is that our finite dispositions cannot determine that we use that procedure since not every application

\footnotetext{
${ }^{7}$ It is natural to choose operators in a natural deduction system, given Tennant's project of taking Gentzen-style introduction and elimination rules as the basis for a general account of meaning. If one's position on the meaning of logical operators prohibits a proof-theoretic treatment, though, other terms are surely available to illustrate the point.
} 
of that procedure involves inputs of a size that agents like us can handle. Therefore, the meaning sceptic gains yet another foothold.

\section{$5 \quad$ Abstractions and semantic dispositionalism}

That the Blackburn-Tennant-Cogburn approach, unadorned, succumbs to the combination problem and the input problem is troubling because the position expresses an insight worth preserving: that an agent's competence with terms is grounded in dispositions to complete feasible tasks. In $§ 5.1$, a seemingly natural attempt to save the position-one invoking idealizing models - is entertained and ultimately rejected. And starting in $§ 5.2$, a more promising solution is offered: by invoking abstracting models, we can (on principled grounds) ignore the traits responsible for an agent's finite limits.

\subsection{Invoking idealizing models}

Consider the following reply to the combination problem and the input problem, couched in terms of the Tennant-Cogburn position. Though this brand of semantic dispositionalist grounds the meanings of one's words by appeal to an ordinary agent's factorizable dispositions to complete simple tasks, so the reply goes, a certain amount of idealization is also required. ${ }^{8}$ Idealizing models impute to a system some trait that it does not actually possess, and in the case of a finite agent, we should expect to idealize away from those traits responsible for her finite limits. ${ }^{9}$ Applied to the combination problem, though an actual agent does not have the traits responsible for possessing all of the combinatory dispositions indicative of adding, an idealized version of the agent, with an unlimited lifespan, memory, and attention span is not similarly hampered. And as for the input problem, while an actual (finite)

\footnotetext{
${ }^{8}$ Tennant does, in fact, make use of idealizations in a related context (1997, 143-150). He does not, however, bring it directly to bear on the problem of immediate interest.

${ }^{9}$ In a certain respect, this approach, employed in reaction to the finitude problem, fits well with the practices of linguists. As Chomsky puts it, "Linguistic theory is concerned primarily with an ideal speaker-listener... who knows her language perfectly and is unaffected by such grammatically irrelevant conditions as memory conditions, distractions, shifts of attention and interest, and errors." $(1965,3)$. Though not the immediate aim of this article, it is worth entertaining how the case made in this article might impact the starting point for linguistics. To that end, see also Cogburn's (2004) reassessment of the significance of behavioral dispositions for current debates in linguistics.
} 
agent cannot ever hope to handle enormous inputs involved in the applications of some terms, the same need not be said for an appropriately idealized version of the agent.

These replies suffer, however, from the same fatal flaw as any appeal to idealizing models on behalf of the semantic dispositionalist, what will be called the multiplicity problem. The traditional form of the concern is that there are multiple ways to idealize the traits of an actual agent, and the only way to privilege one of those idealized versions as the one that fixes what the actual agent means by her words is to assume that which we aim to establish: namely, that she intends addition (rather than quaddition) by ' + '. Hence, the semantic dispositionalist's appeal to idealizations is viciously circular. ${ }^{10}$ Since the multiplicity problem applies to any given trait, it is plain that the situation is no different when idealizing those traits that limit one's ability to combine simple tasks and those traits that limit one's ability to handle inputs because of their size. As such, an appeal to idealizing models does not aid this brand of semantic dispositionalism in answering either the combination problem or the input problem.

\subsection{Invoking abstracting models}

In the next several sections, an alternative reply to the combination problem and the input problem is advanced, building on the case that Jones and I made for the use of abstracting models in defense of semantic dispositionalism (Podlaskowski and Jones, 2012).

To strike the principal contrast between abstracting and idealizing models, consider first the distinction made by Frederick Suppe (1989) between physical parameters and physical quantities: whereas physical parameters are kinds of traits that entities or objects might possess (e.g., mass), physical quantities are amounts of physical parameters (e.g., 412 grams of mass). ${ }^{11}$ Against this backdrop, abstracting from a physical system is understood, for some physical parameter, as not assigning it any physical quantity whatsoever (or, equivalently, not including the parameter in the model at all) - in short, to ignore the parameter altogether. For example, an abstraction from

\footnotetext{
${ }^{10}$ See Kripke $(1982,28)$ and Kusch $(2005,163)$ for related formulations of this objection to the use of idealizations.

${ }^{11}$ Similar distinctions between idealizing and abstracting are prominent in the literature, including those provided by Cartwright (1989), Chakravartty (2001), Ducheyne (2007), Jones (2008), Liu (1999), and Suppe (1989).
} 
an actual pendulum might fail to include mention altogether of the actual temperature of pendulum. The reason for doing so, of course, is that the temperature of the pendulum is irrelevant to its behavior qua pendulum. In contrast, idealizing a physical system is a matter of replacing some physical quantity in that system with a quantity that the system does not actually have. For instance, one way (among many) of idealizing a pendulum is to replace the physical quantity characterizing the finite extension of its bob with a value of zero. In short, what distinguishes abstracting from idealizing models is how physical quantities are handled.

Jones and I have argued that abstracting models better serve the semantic dispositionalist than idealizing models. According to this approach, that an actual (finite) agent means addition by ' + ' is fixed by appeal to the dispositions (to add) of an abstracted version of the agent who does not suffer from the same finite limits as the actual agent. This approach resembles the use of idealizations insofar as the finitude problem is dodged by appealing to a model (of the agent) who is not similarly limited. The two sorts of models differ, though, in that the problem for invoking idealizations begins with there being too many idealizations from which to choose (the multiplicity problem); but with abstractions, no such problem arises. That is, though there are many physical quantities that can replace the one possessed by an actual agent (in an idealizing model), there is only one way to ignore such a quantity (in an abstracting model). Where the physical parameters are traits responsible for our finite limits, abstracting away from those traits can only be achieved in one way, yielding one model. So, the semantic dispositionalist can avoid the finitude problem by abstracting away from those traits responsible for an agent's finite limits (much as for idealizations), and do so without facing the same charge of circular reasoning.

This is not to say, however, that the abstracted version of an agent possesses all of the same dispositions as the actual agent-just those grounding one's competence with a term (what will be called competence-constituting dispositions). More specifically, Jones and I have invoked a distinction between those traits that contribute to one's competence with a term and those traits that contribute to one's performance in its use (Podlaskowski and Jones, 2012, 174-175). The idea is that, because the appeal of abstracting models is to establish the dispositions in virtue of which an actual agent is competent with a term, only traits relevant to said competence should be included in the model. Since the traits responsible for our finite nature (such as our memory capacities) do not appear to be relevant to our possession of 
competence-constituting dispositions - but rather contribute to an account of our performance in using terms - those traits should not be included in the model. As for deciding which traits should be ignored, it is assumed here that the matter is amenable to empirical investigation, where scientists can distinguish in a principled way between traits relevant to one's competence with any given term without presupposing the meaning of any particular one. $^{12}$

The use of abstracting models makes for a natural fit with the BlackburnTennant-Cogburn approach, especially given that such an approach also focuses on those dispositions relevant to one's competence with words. ${ }^{13}$ On such a merged view, what is important for fixing that an actual agent means addition by ' + ' is there being an abstracted version of the agent who possesses the same factorizable competence-constituting dispositions as the actual agent. As for the traits responsible for the special cases of the finitude problem (addressed in more detail in $\S 5.3$ and $\S 5.4$ ), the corresponding physical parameters are not assigned quantities - we ignore them, since they are irrelevant to one's competence with words.

Notice, though, that abstracting away from traits relevant only to one's performance in using a term (such as memory capacity) does not entail that the abstracted agent does not possess those traits altogether. Failure to appreciate this point rests on a confusion between abstractions and idealizations: ignoring some physical quantity is not the same thing as (idealizing and hence) replacing the value assigned to the parameter with a quantity of zero (or any other quantity, for that matter). For example, classical mechanical methods of planetary motion ignore traits such as the particular temperatures of planets, because one's understanding of the system does not require this additional information-but in doing so, no supposition is made that planets fail to have any temperatures whatsoever. Similarly, rather than

\footnotetext{
${ }^{12}$ Jones and I have argued that, while idealizations have been criticized for how they model traits, a parallel criticism does not arise for settling which traits to include a model (Podlaskowski and Jones, 2012, 169). For the use of abstractions, concerns about indeterminacy arise neither for saying how to model a trait nor saying which traits to include in a model $(2012,175-176)$. Also see our discussion of choosing which traits to include in the model, based on empirical grounds (2012, 172 and 175-176).

${ }^{13}$ Tennant invokes a distinction between competence and performance using the nature of computation as his principal guide (1997, 133-137). But while his position on factorizable dispositions is compatible with invoking abstractions, it remains to be established that abstractions prove compatible with Tennant's full-fledged (semantic anti-realist) position. For present purposes, we may remain agnostic on the matter.
} 
assign to the physical parameter of memory capacity a particular quantity that the actual agent does not possess (as one would in an idealization), an abstraction does not include any value whatsoever. Another (perhaps less contentious) way to think about this is that, by not assigning a quantity to such traits, we are abstracting away from the limits of those traits (Podlaskowski and Jones, 2012, 175). Either way, the abstracting model, in effect, provides a sense for what competence-constituting dispositions an agent possesses.

In the next two sections, it is demonstrated how abstracting models feature in replies to the combination problem and input problem.

\subsection{A reply to the combination problem}

Recall the combination problem: our finite limits block our possessing combinatory dispositions to the extent required to fix what we mean by our words. Dispelling this concern requires attending to the traits responsible for own combinatory dispositions and how those dispositions fare in an abstracting model. What the actual agent and the abstracted version have in common are the traits relevant to their (factorizable) competence-constituting dispositions, which includes the combinatory ones; where they differ is that, in the model, the traits irrelevant to those dispositions are ignored. Moreover, the traits responsible for the limits on competence-constituting dispositionstraits such as memory capacity and attention span - are most likely irrelevant to semantic competence (as suggested in §5.2). As such, those traits deserve to be ignored in the model of an agent; and so, in the model, the limits associated with those traits do not affect the competence-constituting dispositions of the abstracted version of an agent. Hence, the dispositions-including the combinatory variety - of the abstracted version of an agent are not limited (by irrelevant and finite traits) so as to invite the combination problem.

That the agent in the model possesses the combinatory dispositions indicative of addition - instead of some quaddition-like counterpart, credited to the ingenuity of the reader - is settled by materials already in place. ${ }^{14}$ Key to establishing that the agent in the model (and by association, the actual agent) is disposed to add is that the relevant combinatory dispositions

\footnotetext{
${ }^{14}$ The mention of quaddition-like possibilities here is reminiscent of the concern raised for fixing our meaning-constituting dispositions by appeal to those possessed by a machine program (Kripke, 1982, 32-35). The account provided here for why agents are disposed to add equally applies to this other objection.
} 
are factorizable, just like dispositions to produce a sum within any given column of an addition problem. As such, the relevant combinatory dispositions constitute the basic sorts of coordinating tasks between columns in the order indicative of addition. Accordingly, the agent in the model (for whom the limits of the actual agent are ignored) is disposed to act in the same way within any given column and in the same way (and order) moving from column-to-column. That is, for an agent without limits, the specified factorizable dispositions yield the set of dispositions indicative of addition.

To Blackburn's position, Kusch raises an objection that resembles the combination problem:

Blackburn's argument makes the questionable assumption that performing a calculation again and again will inevitably push me towards the right result. Alas, there is no inevitability here at all. It could well be the case that the reliability of the result decreases as I check my result repeatedly. $(2006,113)$

This objection takes a broader swipe at Blackburn's position than just his reply to the finitude problem. It also targets Blackburn's reply to the complaint that semantic dispositionalism cannot account for the possibility of error. $^{15}$ For present purposes, though, the focus remains on the finitude problem.

Just as with the combination problem, invoking abstracting models proves helpful. For even though an actual agent might exhibit different dispositions as she repeatedly manipulates the same symbols (as Kusch suggests), the abstracted version of an agent - for whom we have ignored the limit on traits such as continuous attention span - does not obviously suffer from a similar shift in dispositions. This is because the traits responsible for such a shift (in Kusch's example) just happen to be those ignored in the model (for reasons cited in $\$ 5.2$, above). After all, the traits to which Kusch alludes (in his objection to Blackburn's position) are those which are responsible for agents like us possessing finite dispositions - the same traits that motivate the finitude problem. In short, for an abstracted version of an agent, there is no obvious concern that she might possess different dispositions depending on the number of times that she manipulates a symbol.

\footnotetext{
${ }^{15}$ Paul Coates (1997) provides an interesting defense of a Blackburn-style approach to this charge that semantic dispositionalism cannot accommodate the possibility of an agent making mistakes.
} 


\subsection{A reply to the input problem}

Recall the input problem: there are terms (such as 'and') for which some (or perhaps many) applications cannot be broken down into tasks involving manageable inputs of the sort required by the Blackburn-Tennant-Cogburn approach. Much as for the combination problem, invoking abstracting models here proves to be invaluable. In the model of an agent, we ignore those physical parameters of the actual agent that impose a restriction on the size of the inputs involved in the application of any given term. The rationale for doing so is that the traits motivating the input problem, like other traits motivating the finitude problem (and its variations), are irrelevant to one's competence with a term and, therefore, deserve to be ignored in the model.

To illustrate this point, consider again the introduction and eliminations rules for the conjunction operator and how the relevant traits fare in an abstracting model. In the model, we are not imputing to an agent the disposition to handle conjuncts of enormous length (as we might in an idealizing model). Instead, those traits pertaining to handling the length of conjuncts (e.g., working memory, attention span) are ignored, since they are irrelevant to one's competence with the introduction and elimination rules. While Tennant insists that we should only expect of an agent to complete any given step of a proof, the appeal to abstracting models makes sense of agents doing so even if those individual steps involve premises too long and not further factorizable. This roughly matches up with the actual practice of completing complicated proofs, in that for such cases, we regard the lengths of the sentences involved to be irrelevant to the meaning of the conjunction operator.

To Tennant's position, Kusch raises an objection that resembles the input problem:

Tennant's answer to the finitude problem is obviously unsatisfactory. My dispositions to give sums in reply to plus-queries with non-enormous numbers cannot show that I have dispositions to give sums in reply to plus-queries with enormous numbers. (2006, 279 , note 63)

On one reading of this objection, Kusch raises traditional doubts about finite dispositions against Tennant's position. So understood, the objection fails to connect, because Tennant's point is that we should reject the original motivation for the traditional formulation of the problem. But on another 
reading of Kusch's objection, where the concern resembles the input problem, the objection deserves our attention.

We should expect, much as for the input problem, that Tennant cannot help himself to idealizing models in order to dodge the charge. (Kusch rightly denies Tennant such a move.) However, invoking abstracting models is effective in countering this charge. The charge proceeds from the assumption that we cannot establish one's dispositions involving (e.g.) adding together enormous numbers on the basis of those dispositions to add together non-enormous numbers. But by invoking an abstracting model, the traits responsible for our limited ability to handle large inputs are ignored.

In summary, neither the combination problem nor the input problem is a cause for concern, since the traits responsible for raising those problems are irrelevant to what one means, and therefore deserve to be ignored when constructing the appropriate abstracting model. The resultant position is one according to which what an agent means by a term is established by the factorizable dispositions possessed by the abstracted version of the agent.

\section{$6 \quad$ An objection to using abstractions}

The original case made for invoking abstracting models was in reaction to the multiplicity problem. (The response to that problem, offered by Jones and I, was briefly rehearsed in §5.2.) There is, however, an additional criticism that Kripke raises against invoking idealizations which can be adapted to the use of abstractions - one that is presented in $\S 6.1$, and for which a reply is supplied in $\S 6.2$.

\subsection{The ignorance problem}

Kripke suggests that we do not possess the knowledge required for appreciating what the world (or agents in it) would be like, if an idealizing model (used in defense of semantic dispositionalism) were true. He asks: "How in the world can I tell what would happen if my brain were stuffed with extra brain matter...? ... We have no idea what the results of such experiments would be" $(1982,27)$. As such, there is no clear sense for whether the idealized version of an agent has the same (relevant) dispositions as the actual agent. But if the idealized and actual agent fail to possess the same dispositions (of the relevant sort), there is no good reason to think, for example, 
that the actual agent means to add rather than quadd in virtue of whatever dispositions the idealized version of the agent possesses. ${ }^{16}$

Much as for idealizing models, the worry might be raised that we have no genuine sense for what the world (or agents in it) would be like, according to the abstracted model where the traits responsible for our finite capacities are ignored. As such, there is no good reason to conclude that the abstracted version of the agent possesses the same dispositions as the actual agent. Therefore, the grounds are lacking for the conclusion that the abstracted version of the agent and the actual agent mean the same thing by their words. Call this the ignorance problem for abstractions. ${ }^{17}$

\subsection{A reply to the ignorance problem}

The adapted objection, however, is not as convincing as the original one. According to Kripke's criticism, there is no guarantee that an actual agent and a highly idealized one sufficiently resemble each other. If the comparison between the actual agent and the agent in the model were conducted according to their total dispositional states, the differences between them would be apparent, lending further support to the ignorance problem. But by abstracting away from factorizable dispositions, the resemblance between an actual agent and her abstracted counterpart becomes obvious, for they possess the same such dispositions. Indeed, in the case of an actual agent and her abstracted version, they cannot differ in the factorizable dispositions grounding their competence with terms. To appreciate this point, recall that, while the pertinent abstracting model is designed to ignore those traits irrelevant to one's factorizable (meaning-constituting) dispositions, the model must also include mention of those traits relevant to those dispositions. So,

\footnotetext{
${ }^{16}$ For significant replies to Kripke's objection, see Fodor (1990, 94-5) and Pietroski and Rey (1995). See Kusch (2005) for a thoughtful response to Fodor's case. And see Kowalenko (2009) for a equally thoughtful response to Kusch's case.

${ }^{17}$ An alternative reading of Kripke's charge $(1982,27)$ is that the appeal to idealizations is at odds with the expectation that we can settle a priori what we mean by our words. Whether this objection can be answered rests on providing an account of the epistemology of meaning (with, perhaps, an emphasis on introspection) as it pertains to the Blackburn-Tennant-Cogburn approach augmented with the use of abstracting models. Such an account falls well outside the scope of this essay. Still, if any version of semantic dispositionalism proves compatible with a priori knowledge of what one means by their words, a dual emphasis on factorizable dispositions and abstracting models might show a great deal more promise than other varieties of dispositionalism.
} 
if one's factorizable dispositions depend on possessing a certain set of traits, and both the actual agent and the agent in the model possess those traits, they both possess the corresponding factorizable dispositions. By design, then, the agent in the model and the actual agent share the same factorizable dispositions. Since those dispositions fix what one means by a term, the abstracted version and the genuine article mean the same thing by their words. So, contra the ignorance problem, even if we did not know entirely what the world featured in the model would be like, we do have a clear sense for the sorts of meaning-constituting dispositions that would be possessed by the agent in the model. ${ }^{18}$

This response to the ignorance problem for abstractions assumes a great deal about ourselves; establishing the resemblance between an actual agent and her abstracted counterpart requires distinguishing between those traits pertinent to one's competence with a term and those pertinent to one's performance in its use - a point regularly stressed in $\S 5.2, \S 5.3$, and $\S 5.4$. The concern might be raised, though, that we are not the sorts of beings to which such a distinction can be applied. It might be the case that our brains are structured according to an architecture where the traits pertinent to our using a term cannot be separated from those required for competence with the term. Neural networks serve as an apt example, insofar the limits of memory depend upon the number of nodes and their weights. Ignoring the limits on memory here (in an abstracting model), therefore, requires ignoring some of the features constitutive of the neural network. So, if our brains are best understood solely as a neural network, and if meaning-constituting dispositions should be read-off the dispositions of one's brain, it appears that the sought after distinction between competence and performance cannot be applied.

There are empirical grounds, however, for applying this distinction to beings like us. To cite one example, Robert Kowalenko (2009) draws this conclusion based on a survey of recent empirical research on the topic of mental mathematics (one bearing closely to Kripke's concerns about addition). Essential to his case is research that suggests mental mathematics involves several subsystems whose operation can be isolated from the subsystems responsible for, e.g., memory capacity. Insofar as the disposition to

\footnotetext{
${ }^{18}$ If more than one abstracting model of the traits relevant to our meaning-constituting dispositions were available, there might still be doubts about the resemblance between an agent and her abstracted counterpart: it would remain indeterminate whether the resemblance holds. But as already argued by Jones and I (and reconstructed in §5.2), abstracting models do not suffer from the multiplicity problem.
} 
add rests on the interaction between some of the former subsystems, grounds are available for isolating our dispositions to add from other factors such as memory capacity. ${ }^{19}$ To cite another example, Noam Chomsky takes a similar distinction between competence and performance to be "fundamental" to the study of modern linguistics $(1965,4)$. While Chomsky originally invoked the distinction for his theory of syntax, it has since been adapted to the study of semantics - e.g., Cresswell (1978), Larson and Segal (1995), and Chierchia and McConnell-Ginet (2000).

The materials are in place, then, for pinpointing where the proposed counterexample (featuring a neural network) goes wrong. Of course, the brain is a biological neural network involving a number of different subsystems belonging to a nervous system. But the sort of neural network advanced in the example, above, fails to include details relevant to understanding the biological system. The original example focuses only on the nodes of the network and their weights, and the resulting dispositions. While such an approach shows promise when solving certain problems in the field of artificial intelligence, the same characterization of a network is too detail deficient to capture many important features of the brain. Unlike the neural networks featured in the original example, empirical research on mental mathematics suggests that we can isolate subsystems such as those grounding working memory from other subsystems grounding one's arithmetical competency.

In short, there are empirical grounds for the conclusion that we are the sorts of beings for whom the distinction between competence and performance can be applied. This, in turn, provides materials needed for answering the ignorance problem as it arises for abstracting models.

\footnotetext{
${ }^{19}$ Kowalenko's (2009) point is made as part of his defense of idealizations from Kripke's assaults. On the basis of our being able to isolate our dispositions to add from other (interfering) factors, he argues that it is reasonable to allow for casual continuity between the dispositions of actual and idealized objects. While this point is easily generalized to the use of other models in scientific practice, it is unclear whether Kowalenko's case for using idealizations avoids the charge of circularity raised by Kripke. While Kowalenko's principal focus is on Kripke's charge involving the fantastical assumptions made by idealizations, Kowalenko's prospect of addressing Kripke's other charge depends on invoking simplicity considerations which have been brought into question elsewhere (e.g., Kripke 1982, 38; Miller 2002, 10, note 16). Whether or not Kowalenko's use of simplicity ultimately proves problematic, though, the appeal to abstracting models does not involve making the same sort of move.
} 


\section{Conclusion}

In summary, the basic shape of a solution to the finitude problem is established here. The Blackburn-Tennant-Cogburn approach seems to get this much right: we can only expect of an agent that they be competent with terms on the basis of dispositions to complete feasible tasks. But for all of its appeal, this approach faces two special cases of the finitude problem. The motivating insight of the position is preserved, though, by invoking abstracting models. The picture presented here - the result of fusing the two approaches - provides the basic framework for saying how a finite agent can intend a meaningful expression with indefinite (or even infinite) applications, and it does so by permitting itself only naturalistically respectable materials. Not surprisingly, then, the position passes off settling some details to empirical inquiry; but here the basic materials required to answer the meaning sceptic have been put in place. It remains to supplement the present account so as to avoid other traditional objections to semantic dispositionalism.*

\section{REFERENCES}

Blackburn, S. 1984, "The Individual Strikes Back", Synthese 58, pp. 281-302

Boghossian, P. 1989, "The Rule-Following Considerations", Mind 98, pp. 507-549

Cartwright, N. 1989, Nature's Capacities and their Measurement, Oxford: Clarendon Press

Chakravartty, A. 2001, "The Semantic or Model-Theoretic View of Theories and Scientific Realism", Synthese 127, pp. 325-345

Chierchia, G. and McConnell-Ginet, S. 2000, Meaning and Grammar: An Introduction to Semantics, second edition, Cambridge: The MIT Press

Chomsky, N. 1965, Aspects of the Theory of Syntax, Cambridge: The MIT Press

${ }^{*}$ I am grateful to Joseph Baltimore, Nicholaos Jones, William Melanson, Neil Tennant, Joshua Smith, and four anonymous referees for providing extremely helpful comments leading to the final version of this article. 
CoAtes, P. 1997, "Meaning, Mistake and Miscalculation", Minds and Machines 7 , pp. 171-197

Cogburn, J. 2004, "Inferentialism and Tacit Knowledge", Behavior and Philosophy 32, pp. 503-524

Cresswell, M.J. 1978, "Semantic Competence", in: F. Guenthner and M. Guenthner-Reuter, eds., Meaning and Translation. London: Duckworth

Ducheyne, S. 2007, "Abstraction vs. Idealization”, The Reasoner 1(5), pp. 9-10

Fodor, J. 1990, A Theory of Content and Other Essays, MIT Press: Cambridge

Haukioja, J. 2004, "Kripke's Finitude Objection to Dispositionalist Theories of Meaning", in: J. Marek and M. Reicher, eds., Experience and Analysis. Papers of the 27th International Wittgenstein Symposium. Kirchberg am Wechsel: Austrian Ludwig Wittgenstein Society

Jones, N. 2008, "Is All Abstracting Idealizing?", The Reasoner 2(4), pp. $4-5$

KrIPKe, S. 1982, Wittgenstein on Rules and Private Language, Cambridge: Harvard University Press

Kowalenko, R. 2009, "How (Not) To Think About Idealisation and Ceteris Paribus-Laws", Synthese 167, pp. 183-201

Kusch, M. 2005, "Fodor v. Kripke: Semantic Dispositionalism, Idealization and Ceteris Paribus Clauses", Analysis 65(2), pp. 156-63

Kusch, M. 2006, A Sceptical Guide to Meaning and Rules, Ithaca: McGillQueen's University Press

Larson, R. and Segal, G. 1995, Knowledge of Meaning: An Introduction to Semantic Theory, Cambridge: The MIT Press

LiU, C. 1999, "Approximation, Idealization, and Laws of Nature", Synthese 118, pp. 229-256 
Martin, C.B. and Heil, J. 1998, "Rules and Powers", Noûs 32(12), pp. $283-312$

Miller, A. 1997, Philosophy of Language, London: UCL Press

Miller, A. 2002, "Introduction", in: A. Miller and C. Wright, eds., RuleFollowing and Meaning. London: UCL Press, pp. 1-15

Petтit, P. 1999, "A Theory of Normal and Ideal Conditions", Philosophical Studies 96, pp. 21-44

Pietroski, P. and Rey, G. 1995, "When Other Things Aren't Equal: Saving Ceteris Paribus Laws from Vacuity", The British Journal for the Philosophy of Science 46(1), pp. 81-110

Podlaskowski, A. 2010, "Reconciling Semantic Dispositionalism with Semantic Holism", Philosophia 38, pp. 169-178

Podlaskowski, A. and Jones, N. 2012, "Idealizing, Abstracting, and Semantic Dispositionalism", European Journal of Philosophy 20(1), pp. $166-178$

SprevaK, M. 2008, "Kripke's Paradox and the Church-Turing Thesis", Synthese 160, pp. 285-295

Suppe, F. 1989, The Semantic Conception of Theories and Scientific Realism, Chicago: University of Illinois Press

Tennant, N. 1997, The Taming of the True, New York: Clarendon Press 